EGU21-16407

https://doi.org/10.5194/egusphere-egu21-16407

EGU General Assembly 2021

(c) Author(s) 2022. This work is distributed under

the Creative Commons Attribution 4.0 License.

\title{
Estimation of the electric field and the altitude from spectrophotometric observations in limb-viewing geometry
}

\author{
Matthieu Garnung ${ }^{1}$, Sebastien Celestin ${ }^{1}$, and Thomas Farges ${ }^{2}$ \\ ${ }^{1}$ LPC2E, University of Orleans, CNRS, France \\ ${ }^{2}$ CEA, DAM, DIF, F-91297, Arpajon, France
}

Sprites are bright and sudden events occuring above thunderstorms between 40 and $90 \mathrm{~km}$ altitude. These phenomena are usually observed using ground-based cameras and from spacecrafts. The Imager of Sprites and Upper Atmospheric Lightning (ISUAL), a payload on the FORMOSAT-2 satellite, recorded several sprite events during its mission. Contrary to JEM-GLIMS (JAXA) or ASIM (ESA), which are space missions dedicated to the observation of TLEs from a nadirviewing geometry, ISUAL used a limb-viewing geometry. This configuration offers the possibility to directly estimate the altitude of the event from its camera.

The challenge consists in estimating the altitude and the electric field from spectrophotometer measurements. The method of the spectrophotometric ratios consists to use ratios computed from different band systems to estimate the altitude and the electric field. It is the one of the most encouraging to achieve this goal.

In this work, we propose a method to estimate the electric field and the altitude from an observation made by the ISUAL instrument using the following ratios LBH/1PN2, 2PN2/1PN2 and $\mathrm{LBH} / 1 \mathrm{NN} 2+$. We show that some spectroscopic ratios are more useful then others and point out some limitations of this approach that will need to be widen to nadir-viewing geometry observations. 